\title{
5
}

\section{Managing Pediatric Pain at School}

\author{
Ronald T. Brown
}

\begin{abstract}
Summary
This chapter reviews literature pertaining to the management of pain within the school setting. Etiological issues underlying obstacles to school attendance are reviewed, including issues pertaining to make-up work, concerns among many children pertaining to use of the bathroom at school, diet and eating habits at school, relationship with a teacher or peer, fear of pain episodes at school, learning problems, test or performance anxiety, separation anxiety, and familial reinforcement of sick behavior. Interventions to increase school attendance are reviewed; these also include a careful assessment of the child and the family system, the use of behavioral interventions, as well as other treatment approaches, including the use of relaxation therapy and problem-solving therapy. Finally, specific directions for future research efforts and training also are provided.
\end{abstract}

Key Words: Anxiety; pain; pediatric; recurrent abdominal pain; school.

\section{School as a Venue for Health Care}

Reforms in health care have resulted in increased emphasis on specific access to care and the reduction of costs. One of the pathways of shifting health-related services is from tertiary care centers to primary care and community settings (1). Because existing mechanisms in schools can serve children and adolescents with special needs, schools have been identified as a viable option for placement of community health care services. As Power and colleagues observed, schools are uniquely positioned to assist in the management and prevention of children's health problems (2).

Several other factors support the change in focus of health care delivery from tertiary care settings to community settings (1). These include advances in child development that underscore a social-ecological model by attending to all systems in the child's life (e.g., home, school). Moreover, the limitations of the medical model that traditionally has assumed a deficit model in health care have

From: Bringing Pain Relief to Children: Treatment Approaches

Edited by: G. A. Finley, P. J. McGrath, and C. T. Chambers $\odot$ Humana Press Inc., Totowa, NJ 
become increasingly apparent. Prevention, resilience, and positive adaptation have become the mainstays in health care. In support of this change, a number of interventions use elementary schools to address prevention of injury, including playground safety, seatbelt safety, fire safety, avoidance of spinal cord injuries, home safety programs, child sexual abuse programs, drug abuse education, driver education, and school violence (3).

School interventions offer opportunities like easy access to assessment data, a venue to examine specific functions of behavior, the possibility of multidisciplinary teams for collaboration on assessment and remedy of specific behavior, and access to multiple change agents (e.g., peers, teachers) (1). School personnel are ideally positioned for intervening directly in the child's natural environment, monitoring interventions in this environment, and providing a context for observing how competent, healthy children develop.

As noted, the social-ecological model underscores the importance of integrating systems of care, including the school. Child competencies and behaviors are observed not only at home but also at school. For example, a child with sickle cell disease will show specific symptoms or functional impairments at home and school. Thus, the school is an excellent place to assess impairments and implement intervention efforts. Stressors associated with a disease (e.g., the numerous painful events associated with sickle cell) are frequently manifested at school (e.g., problems with achievement or peer relationships). For disorders with recurrent pain, the stress that occurs at school will likely have an impact on functioning and decrease performance.

As Walker observed, life stress may play an especially crucial role in either precipitating or sustaining pain symptoms (4). For example, Walker and associates noted that children with recurrent abdominal pain reported a greater frequency of daily stressors relative to their healthy peers (5). Such stressors were associated with school-related activities, and disease included difficulty in comprehending homework assignments and excessive worries about examinations and grades. Children with recurrent abdominal pain also reported excessive anxiety and dysthymia on days that they also experienced stressful events. Walker and colleagues observed that these children reacted to stressful events with abdominal pain and other somatic symptoms, and the researchers concluded that children with recurrent pain may have a specific type of stress reactivity that manifests itself with specific somatic symptoms. Whether this is true for children with all types of recurrent pain is unclear. However, the role of stress and how to cope with stress are clearly associated with successful pain management.

Tsao and colleagues examined associations between gender and laboratory pain reactivity to the cold pressor task, a laboratory-placed analog that most closely approximates real pain experiences (6). School absences and self-initiated 
school nurse visits were also examined in 57 children ranging in age from 8 to 10 years. Cold pressor task pain ratings, tolerance to pain, and gender were examined in relation to nurse visits and school absences. The number of nurse visits and school absences were collected over 2 years. Higher pain ratings and female sex predicted more school absences. In addition, female sex also predicted more frequent nurse visits for acute complaints with documented physical findings. Moreover, data suggested that girls are more likely than boys to miss school and visit the nurse for acute illnesses. These findings are important because they suggest the potential for screening children who may be especially vulnerable to frequent school absences based on self-reports of pain. The results from this investigation also indicate that girls may be especially vulnerable to functional impairments (i.e., school attendance) because of recurrent pain.

In a follow-up study to the Tsao et al. investigation (6), Tsao and Zeltzer examined sex differences in self-initiated school nurse visits and pain-associated symptoms in 57 preadolescents aged 9-11 years (7). The preadolescents were asked to complete the pain-focused version of the Children's Somatization Inventory. Pain symptom scores, gender, and the interaction of pain symptoms and gender were examined in relationship to nurse visits that were tracked over 1 year. Neither sex nor pain scores alone predicted total nurse visits. For female preadolescents, higher pain symptom scores predicted total visits for complaints with documented physical findings. This was consistent with the findings of the elementary school sample conducted by Tsao et al. (6). A complex association was demonstrated in this preadolescent sample among gender, pain-related symptoms, and self-initiated nurse visits. Again, the investigators interpreted their data to suggest that pain-focused symptom measures may predict later pain management.

Tsao and Zeltzer suggested that the identification of these symptoms by selfreport measures may be important in predicting health care utilization, particularly for young adults (7). Whether preadolescent pain management or adolescent pain management predicts later coping with pain during young adulthood is an important area of inquiry for future research. Clearly, longitudinal studies will be needed in this area, particularly studies using longitudinal methodology.

Walker and Greene demonstrated that children who experience a number of negative life events are more apt to exhibit pain symptoms over time relative to children reporting few negative life events (8). The researchers hypothesized that these children have difficulty deriving support from others, particularly peers, because they also are involved in fewer peer activities relative to their healthy peers. Walker suggested that interventions designed to assist children and adolescents in increasing social competencies with peers may concomitantly enhance these children's capacity to cope with pain symptoms (4). 
In support of the notion that negative life events or the absence of peer social support may be associated with frequent health symptoms, Williams and coworkers estimated the prevalence of bullying in nearly 3000 elementary schoolchildren in a large urban area in the United Kingdom (9). The investigators examined the association of bullying with common health symptoms in childhood. The dependent measures in the investigation included self-reported bullying and common health symptoms. Approximately one-fourth of the cohort for whom information was available reported that they had been bullied. An association was found between children who reported being bullied and not sleeping well, bed wetting, symptoms of depression, and experiences of minor somatic symptoms, including headaches and stomachaches. There was a positive association between increasing risk of health symptoms and bullying. These data provide compelling evidence for the association between somatic symptoms and peer supports. The investigators cautioned that this association may not necessarily be causal, but they underscored the role of peer support in children's coping with somatic issues. Studies are needed that systematically examine objective measures of peer relationships, including peer sociometry as rated by other peers and the relationship of these assessments to somatic symptoms and coping with pain.

As Walker cautioned, most investigations of children with recurrent pain have been conducted on pediatric patients in tertiary care facilities (4). The pain of these children is likely to be of longer duration and is more likely to be accompanied by significant psychiatric comorbidity, as well as be refractory to traditional treatments, relative to children evaluated and treated in a primary care facility. Walker observed that distress among these patients may be higher than in community or school samples, suggesting that problems identified in the primary care office or school setting may also be identified rather easily and readily managed by caregivers and school professionals. Epidemiological studies are needed that systematically examine potential differences between children and adolescents who are brought to their primary care providers vs children who come to a tertiary care setting.

\section{Obstacles to School Attendance}

Walker delineated several obstacles to school attendance (4). These include problems with make-up work, explanations to teachers and peers regarding why the children may have missed so many days of school, children's reluctance to use bathrooms at their schools, diet and eating habits at school, relationship with a teacher or peers, fear of pain episodes at school, learning problems, test or performance anxiety, separation anxiety, and family reinforcement of sick role behavior. Walker noted that a gradual return may help children return to school full time. Such a schedule may also assist them in eventually regaining 
their confidence that they can survive a pain episode at school. Walker suggested that full-time attendance is typically possible within 3-4 weeks after the implementation of a program designed to help school attendance for children with pain episodes.

\subsection{Make-Up Work}

Many children seem to be overwhelmed by the amount of make-up work when they return to school after a prolonged illness (4). Some children fear that they may not be able to complete all of the work, or that they may not understand the assignments. These feelings of self-doubt and negative cognitions may even exacerbate pain symptoms. In these cases, Walker suggested a structured plan in which a missed assignment is broken into manageable parts, with a schedule that underscores specific progress toward accomplishing missed assignments rather than completing final products. Caregivers and teachers can establish specific periods of time for making up missed assignments (e.g., performing make-up work for approx 30 minutes/day). Frequently, caregivers must contact the school to determine what make-up work will be required and to negotiate a reasonable timeline for completing the assignments. A reduction in work assignments will sometimes be necessary if the child cannot manage the completion of the tasks within a specific time frame.

\subsection{Explanations to Teachers and Peers}

Many children become anxious that teachers or peers may ask them why they have been absent from school, particularly if the illness does not have a specific organic etiology or medical tests may not be conclusive in identifying specific pathology. As Walker suggested, some children may need specific assistance in preparing a response (4). In other cases, it may be helpful to have a letter from the child's primary care provider indicating that the illness is legitimate. The primary care provider could recommend returning to school for only part of the day or using the bathroom whenever the child deems it necessary. Walker et al. acknowledged that, even in the absence of any identifiable organic disease, pain may be as severe and functionally incapacitating as in conditions for which there is identifiable organic pain (10). It is important that professionals communicate an acknowledgment of pain and clarify coping strategies in pain management (4).

\subsection{School Bathrooms}

Many children with gastrointestinal disorders or recurrent abdominal pain may be reluctant to use the bathrooms at their school (4). These children may avoid school bathrooms because of lack of privacy, poor sanitation, lack of toilet paper, or the fear that classmates may know that they are defecating in the 
bathroom. In some cases, children may fear either physical or verbal abuse by other children while in the bathroom. In other cases, children may fear that using the bathroom may take too long and could eventually result in reprimands from the teacher for tardiness. Walker suggested the health care provider write a letter to the school indicating that the child should be allowed to use the bathroom whenever necessary. This would put the timing of bathroom use under the child's control and diminish anxiety about accidents. In addition, it will allow the child to use the bathroom when peers are not present. Parents can advocate for sanitary bathroom facilities in the public schools. Walker also suggested that children learn to use public bathrooms in specific progressive stages, beginning with bathrooms in the homes of friends and relatives and subsequently restaurants and private buildings. The child can be rewarded for using bathroom facilities in new settings, which it is hoped will generalize to school settings and enhance self-competence and adaptation to pain experiences outside the home.

\subsection{Diet and Eating Habits at School}

For children with recurrent abdominal pain, Walker noted that diet and eating habits may exacerbate symptoms (4). Available foods and schedules for school lunch may contribute to symptoms. Many children may not have sufficient time to ingest their food and use the bathroom in the amount of time provided for lunch. In other cases, the types of food and beverages may also increase gastrointestinal symptoms and result in abdominal distress. In such cases, parents can advocate for specialized diets. With the epidemic of obesity in children and adolescents and the resulting increased risk for other diseases (e.g., type 2 diabetes, cardiovascular problems), it would be appropriate for the school to advocate for good nutritional standards in promoting positive health behaviors.

\subsection{Relationship With a Teacher or Peer}

In many cases, children with recurrent pain, particularly recurrent abdominal pain, may have difficulty adjusting to a teacher who is more matter-of-fact or less nurturing than previous teachers, which is sometimes misinterpreted by some children as disapproval $(4,11)$. In such cases, it is useful for the parents to meet with the teacher, principal, or school counselor to get to know the teacher better. In many cases, after frank discussion, the teacher can assist in helping the child to adjust, and the child can better understand the teacher's style.

As Walker asserted, a change of teachers should only be initiated as a last resort (4). Such a change could reinforce beliefs or distortions that the child is unable to cope with. Also, additional stressors might result in adjusting to a new classroom, particularly for a child who already has other stressors and perhaps inadequate coping skills. Some children report taunting or teasing by peers in 
the classroom. In such cases, it is useful for caregivers to meet with school personnel to work jointly in resolving such issues.

\subsection{Fear of Pain Episodes at School}

Children who have poor coping skills for dealing with pain may often harbor exaggerated fears that they will have a pain episode at school and not be able to manage it. Walker observed that such distress arises from an ongoing habit of focusing attention on minor physical sensations and fearing these sensations will increase in intensity (4). This results in noxious sensory experiences, increased anticipatory pain, increased anxiety, physiological arousal, diminished pain thresholds, and increased distress $(12,13)$. This is a cyclical effect in which anxiety about pain diminishes pain thresholds and capacity for coping with pain.

A primary goal in working with these children is to assist them in learning that they can cope with their pain (4). Walker recommended that children's initial return to school be brief, perhaps only 1-2 hours/day. Most children will be able to attend school and even manage their pain for brief periods. The brief school attendance will assist children in building confidence so they may later be able to survive a pain episode at school. The child's health care provider and caregivers should have a plan in place in case the child experiences pain while at school. It is typically best if the child is allowed to lie down and rest until well enough to return to class or until it is time to leave school for the day. A child may also work with a school counselor to learn relaxation or distraction techniques $(4,14)$. Walker cautioned it is often counterproductive for children to call home or be allowed to leave school early when a pain episode occurs as this reinforces complaining and passive coping styles. Walker et al. noted that children who use passive coping strategies like withdrawal (e.g., remaining home from school or returning home quickly after attending school for a short period of time) are more likely to maintain these patterns and symptoms over the long term (5).

\subsection{Learning Problems}

A history of academic difficulties in one or more subject areas, problems with attention and concentration, failure to complete assignments, or an inappropriate class placement may signify a learning problem or a stressful experience. Some children may use pain to cope with such difficulties. To increase coping skills, caregivers may need to contact school personnel to develop a plan that might include special education services, tutoring, or assistance with organizational skills (4). Efforts should be made to minimize the chance of the plan failing, and procedures should be implemented that allow the child to make a smooth transition back to school. 


\subsection{Test or Performance Anxiety}

Many children report a greater frequency of pain symptoms prior to a stressful event or competitive activity at school, including athletic events and examinations (4). Such circumscribed anxiety may benefit from the use of positive self-coaching statements, such as, "I know I can do it if I just do my best." Negative self-statements, such as "What if I fail?," should be eliminated. The use of adaptive self-statements is important to enhance coping strategies and diminish distorted negative thoughts when approaching stressful tasks.

\subsection{Separation Anxiety}

Separation anxiety refers to developmentally inappropriate and excessive anxiety concerning separation from home or from those to whom the child is attached (15). One symptom criterion for the diagnosis of separation anxiety disorder includes "repeated complaints of physical symptoms (such as headaches or stomachaches, nausea or vomiting) when separation from major attachment figures occurs or is anticipated" (p. 113). Typically, such symptoms occur on the morning of schooldays and are less pervasive on weekends when the child will not be separated from caregivers (4). Symptoms may reoccur on Sunday evening in anticipation of separation from caregivers to attend school on Monday.

Other symptoms of separation anxiety include recurrent or excessive distress when separation from home or major attachment figures occurs or is anticipated; persistent and excessive worry about losing, or possible harm befalling, major attachment figures; persistent and excessive worry that an untoward event will lead to separation from a major attachment figure (e.g., getting lost or being kidnapped). Other issues include persistent reluctance or refusal to go to school or elsewhere because of fear of separation, persistent and excessive fearfulness or reluctance to be alone without major attachment figures at home or without significant adults in other settings, persistent reluctance or refusal to go to sleep without being near a major attachment figure or to sleep away from home, and repeated nightmares involving the theme of separation (15).

In accordance with psychiatric criteria, the duration of disturbance must be at least 4 weeks, and the onset must before age 18 (15). There must also be some functional impairment, including clinically significant distress or impairment in social, academic, or other important areas of functioning. In addition, separation anxiety disorder does not occur exclusively during the course of a pervasive developmental disorder, schizophrenia, or other psychotic disorder and, in adolescents and adults, is not better accounted for by panic disorder with agoraphobia.

Often, caregivers of children with separation anxiety disorder have an enmeshed relationship with their children. Children may sleep with their parents and may sit very close to the parents during the interview (4). Both the caregiver 
and the child tend to perceive pain as so severe that it makes school attendance impossible. Because a complete cure for many types of pain is quite difficult to accomplish, caregivers must be convinced that it is necessary to teach their children appropriate coping skills for managing pain. It is important for caregivers to support their children in attending school for at least part of the day despite their children's pain. A primary goal of treatment is to thwart the cycle of the separation anxiety for both the caregivers and the child. Thus, it is recommended that caregivers and their children be allowed initially to limit attendance at school for a specific amount of time so they can be confident of their success in managing pain at school. This period of time will vary depending on the severity of separation anxiety. School attendance must be reinforced by the caregiver, so it is necessary for the caregiver to be comfortable with the child's partial return to school.

\subsection{Family Reinforcement of Sick Behavior}

No specific differences have been found between families of well children and those of children with chronic pain on family measures like marital satisfaction and cohesion (4). However, some clinical evidence suggests that these families may differ in areas not gaged by standardized instruments. Overprotectiveness and enmeshment are two of these areas. Walker suggested that social modeling of pain may contribute to recurrent pain episodes among children, particularly if the child vicariously observes a parent receiving attention from other family members if there are complaints of pain. Levy and coworkers noted that a child may learn pain behavior when caregivers seek extensive medical consultation for pain (16). Walker noted that caregivers of children with abdominal pain may view their children as vulnerable, and these caregivers may attempt to protect their children from potential health-related threats (4). This pattern may result in caregivers letting a child stay home from school because of minor ailments like stomachaches.

Walker et al. noted that absences from school may serve to reinforce a child's pain behavior, particularly when the child is excused from examinations, sporting events, or other activities that involve performance (17). This is especially true for children who fear failure. In addition, the researchers observed that some children may gain social reinforcement (i.e., special attention, privileges) as a result of their symptoms, which in turn may increase sick role behaviors. Walker et al. reported that children with recurrent abdominal pain perceived their caregivers as providing them with greater attention and relief from responsibilities than that reported by other children (10).

Thus, children may gain special attention for sick behavior or their illness. In these cases, it is useful to have specific guidelines (e.g., fever) for caregivers regarding when children should be allowed to remain home from school. The 
child who stays home should be made to remain in bed during the day, eliminating reinforcement of sick role behaviors (4). It is also wise for schools to provide reinforcement for behaviors that are associated with competence, such as school attendance.

\subsection{Summary}

For children with chronic pain, there are a number of common obstacles to school attendance that must be assessed before implementing any treatment program designed to increase school attendance $(4,18)$. These obstacles include children being overwhelmed by make-up work, concerns about explanations to teachers and peers about the pain, concern about the use of school bathrooms, particularly for children and adolescents with recurrent abdominal pain, problems with relationships with teachers and peers, fear of painful episodes at school, presence of learning problems or inappropriate school or classroom placement, test performance or anxiety, separation anxiety, the family's reinforcement of the child's sick role behavior, children's emotional distress, and general life stress. It is imperative that the practitioners evaluate these issues before designing or implementing a plan or program for the child to return to school.

\section{Increasing School Attendance}

\subsection{Assessment}

The most important step in assisting children's return to school is the identification of specific obstacles to school attendance $(4,18)$. The pediatric or mental health provider must conduct a detailed assessment of obstacles to school attendance. Questions about obstacles to school attendance should be nonthreatening and designed to solicit general conversation with the child about school. Typically, conversations with children will provide a great deal of information about school attendance and issues of anxiety. In general, this is a time for the provider to establish rapport with the child. In most cases, it is usually not helpful to ask yes or no questions because open-ended questions will elicit more from the child.

Walker constructed a specific semistructured interview for providers (4). Questions might include the following: What do you like best (least) about school? Which grade do you like better, the one you are in now or the grade you were in last year? What was your favorite year (favorite teacher) in school? Why? What is similar or different about this school year? What will it be like when you go back to school? Do you have a lot of make-up work? What will the kids (teacher) say when they see you? Will the teacher be glad to see you? What will you do if you have a stomachache (or other pain) at school? Will you be able to use the bathroom at school when you need to? Do you sleep in your 
own room at night? Does anyone sleep with you? How do you spend the day when you stay home from school?

Responses to these questions will provide information to the practitioner about obstacles to school attendance and identification of specific stressors surrounding school attendance. After determining the stressors, the provider can work with the family and school personnel for interventions that improve school attendance. It is especially important to work with children on school-related issues in anticipation of future medical regimens, coping with returning to school, managing peer questions, or concerns about disfigurement (e.g., in the case of burns).

A careful analysis is critical in the identification of factors that may contribute to the child's or the family's reluctance about the child's return to school. These data should be elicited from both the child and the caregiver in a forthright interview, with specific questions about coping with return to school, managing peer questions, and concerns about a new school environment.

\subsection{Interventions}

Walker recommended that a graduated plan be implemented that incorporates as its ultimate optimal goal returning to school full time (4). This is suggested because some children, particularly those who have separation anxiety, will become distressed at any mention of returning to school. Walker acknowledged that, if children with recurrent pain are required to attend school the day following their medical evaluation, it is likely that they may manifest a severe pain episode that will serve to prevent or impede school attendance. As noted, an important first step in assisting children in their return to school is the identification of obstacles and addressing these specific issues and concerns $(4,18)$. Walker also suggested that the health care provider consult with both the child and the caregiver to identify a manageable goal for initial attendance at school. The provider generally begins with the goal of a half-day return to school. If the child or caregiver notes concern, the time might be decreased until both the child and caregiver are confident that the child will be able to succeed.

In developing any type of intervention program, the health care provider should contract the child's caregivers to determine their motivation. Also, caregivers need to acknowledge they will be amenable to obtaining additional assistance if the child is not successful in the treatment program (4). Typically, a child should be successful in returning to school at least within 3 weeks after commencement of the program.

\subsubsection{Behavioral Approaches}

It is recommended that a program be implemented that reinforces school attendance. Such an approach might include a token economy system in which the child receives ongoing reinforcement for progress in attending school. For 
example, the use of small incremental periods of school attendance might be reinforced with either special privileges or small rewards. Points might be earned and eventually traded for larger prizes. As Walker noted, it is important to consider the specific characteristics of the child and the family and to use a reward system that is sufficiently reinforcing to the child (4). Also, make sure the family is capable of implementing the system. It is critical that returning to school be a family affair, with all family members in agreement on the contingencies and the reinforcers. The family must be sufficiently organized to apply the reinforcement in a timely and consistent manner immediately after the desired behavior of school attendance is achieved. The system of rewards should be motivating enough that the child is interested in receiving additional rewards, and full school attendance will eventually be possible. The rewards may be increased or decreased on a variable schedule. Additional reinforcers may also be provided to increase other desired behaviors (e.g., not crying when returning to school). Verbal praise should always be provided in addition to tangible rewards. Further, as children lose interest in the current rewards, a system should be in place for new rewards that are meaningful and desirable for the child and will also motivate desired behavior.

Ultimately, a system might be used in which the child could trade points for larger rewards over a longer period of time. Eventually, longer-term goals can replace shorter-term goals until the eventual goal of returning to school full time is accomplished. For issues of separation anxiety, children may have more difficulty in separating from one parent than another. In such situations, the parent from whom it is easier to detach should bring the child to school. Review the behavioral program on an ongoing basis to evaluate its efficacy and to develop additional long-term goals (4).

\subsubsection{Other Treatment Approaches}

Other treatment approaches have been evaluated for children and adolescents with recurrent pain. These approaches have primarily involved relaxation training designed to diminish frequency and intensity of pain $(14,19,20)$. Larsson and Carlson discussed the advantages of treatment programs in schools for children and adolescents with chronic pain (14). School health professionals (e.g., a school nurse, school psychologist) who are available to children during school hours and between training sessions can manage such programs. When such approaches are administered at school, they may be potentially offered in a group format, thereby serving as many children and adolescents in need as possible. Implementation of such programs at school enhances the possibility of external validity or generalizability. Such approaches are cost-effective and may be implemented by school personnel. 
Larsson and Melin conducted an experimental study of 32 adolescent high school students who ranged in age from 16 to 18 years (19). All of the adolescents were identified with various types of chronic headaches, including tension and combined tension and migraine headaches. All of the participants were treated in school. Participants were assigned randomly to a nine-session relaxation training program or to an information-contact condition. Both of these treatment conditions were contrasted to a no-contact control condition. Relaxation therapy alone resulted in the reduction of headache complaints. Participants at their 6-month follow-up evaluations continued to have fewer complaints. The effects obtained in the information-contact showed only minor improvements relative to the relaxation condition. Although this investigation did not lend itself to issues of separation anxiety or returning to school, the findings are important because they demonstrate the efficacy and success of a pain intervention program at school.

Larsson and associates conducted a controlled trial in which the efficacy of a self-help relaxation approach was compared with problem discussion and a self-monitoring condition (20). Participants were 36 high school students who suffered from tension and combined tension and migraine headaches. The students were treated during a 5-week period in a traditional school system. The self-help relaxation treatment condition resulted in improvements on all dimensions of the participants' headaches. Conclusions from the investigation were that self-help relaxation training is a promising, cost-effective procedure to manage chronic headaches in adolescents. These investigators demonstrated the viability of such an intervention in a high school setting.

In a more recent investigation, Larsson and Carlsson compared the efficacy of a school-based, nurse-administered relaxation training intervention with a no-treatment control condition for 26 children and adolescents ranging in age from 10 to 15 years (14). All of the participants in the investigation suffered from chronic tension headaches. Participants were assigned randomly to either the relaxation intervention or the no-treatment control group. They were assessed pre- and posttesting and at a 6-month follow-up. Headache activity for the children treated with relaxation training was significantly reduced relative to the control condition at both posttreatment and the 6-month follow-up. Specifically, 69 and $73 \%$ of the children and adolescents at posttest assessment and at 6-month follow-up, respectively, who were treated with relaxation had achieved clinically significant headache improvement (50\% improvement) relative to $8 \%$ and $27 \%$ of the pupils in the control group, respectively. This investigation underscored the viability of such a treatment program in a school setting for children and adolescents with recurrent pain. 


\subsection{Summary}

It is important to assess whether a family is organized, capable, and sufficiently motivated to implement a behavior management plan. A plan that emphasizes return to school on a graduated basis is generally most acceptable and effective, with the ultimate goal full return to school. Typically, such a plan takes approx 3 weeks to complete. If the child has not returned to school full time in the selected time, it is recommended that a mental health care provider be consulted for assistance. The initial goal for return to school should be made easy for the child and family to accomplish (e.g., returning to school for a portion of the day). Rewards for school attendance should be within the family's budget, and the rewards should be provided as soon as they are earned and not be available to the child by other means. Rewards can consist of small prizes, special activities, or stars that can be redeemed for other prizes or activities. The family also should be provided with a copy of the behavior plan reward schedule. The school should also be aware of the behavior plan for returning to school.

When rewards are given, parents should also provide verbal praise. Rewards should be given for each period of school attendance but should diminish over time as the behavior is more concretely in place. It is important that all caregivers be involved in the program, and that the program be reviewed on a regular basis for revision and to reflect accomplishments, new goals, and new rewards. Long-term goals should include sustained school attendance.

Few studies have examined interventions designed to manage recurrent pain in the school setting. There are a number of advantages to school-based interventions, including availability of personnel, access to many children, generalizability, and cost-effectiveness. The few studies that are available suggest the potential efficacy of these programs for managing recurrent headaches in the school setting. More clinical trials are needed and should involve the child, the school, and the family in management of recurrent pain.

\section{Conclusions and Future Directions}

Recurrent pain in children and adolescents represents a major public health concern. Concomitantly, numerous changes have permeated the delivery of health care services in the United States, including the provision of services at the level of primary care (21). The result has been a trend toward placing systems of care for pediatric populations into schools and a trend away from provision of services at the level of tertiary care centers. The school further represents a venue where additional disease prevention and health promotion can occur. Pain management may take place in schools, coping skills may be fostered, and partnerships may be forged among pediatricians, families, and mental health providers. 
The importance of careful and ongoing assessment to identify potential barriers to school attendance must be underscored, particularly as these barriers are associated with recurrent pain episodes. Semistructured, structured, and paper-and-pencil self-report interview procedures should be used to identify barriers. Unfortunately, there is insufficient psychometric data on assessment techniques used in schools. The development and validation of assessment instruments are necessary, especially measurements designed to evaluate recurrent pain and how stressors associated with school attendance affect children's management and coping.

This chapter underscores the potential efficacy of behavioral approaches in supporting children's return to school. Numerous reasons for lack of school attendance are reviewed, including separation anxiety, fear of returning to school because of missed work, specific health- and pain-related issues that are made worse in the school setting, and inappropriate school placement. Despite the undisputed efficacy of behavioral approaches at school, potential barriers exist. These can include a lack of information among both caregivers and school professionals on the value of behavioral approaches. Other barriers that might impede implementation of these behavioral techniques include severe psychopathology of caregivers, lack of motivation on the part of caregivers and school personnel, and reinforcement systems that are not sufficiently motivating to the child or are not followed carefully.

Other therapeutic approaches like relaxation techniques and cognitive behavioral interventions given at school show promise. Such approaches may be applied to many children simultaneously. They are especially ecologically valid and generalizable and are cost-effective because they can be conducted by professionals already employed in the school system.

Much of what has been reviewed in this chapter points toward recommended changes in public policy for children who experience chronic pain and at the same time attend school. We anticipate that public policy ultimately will be expressed through federal and state legislation that it is hoped will dictate appropriate allocation of resources that allow for the incorporation of pediatric psychological and mental health services in schools. Many complex services will be needed for schools and the families served by them, including an increase in medical, educational, and psychological services. Perrin and Ireys observed that the organization of services for children with chronic illnesses is both diverse and fragmented (22). A first effort and necessary first step will be to integrate pediatric and psychological services across multiple locations, including schools and pediatric health care settings.

Thompson and Gustafson underscored that a major source of stress in caring for children with chronic disease is economic (23). Although efficiency in the provision of services is important, direct costs of services (e.g., mental health 
staff services, adjunctive care for families, and transportation for caregivers) will also be important. Legislation for specialized services to be provided to children and adolescents with recurrent and chronic pain can and should be advocated by the national associations representing pediatrics, psychology, and psychiatry.

Training efforts need to continue to focus on health care providers and the appropriate delivery of services in schools. Efforts need to be made to train school personnel, including school nurses and school psychologists, in the appropriate delivery of specialized pediatric psychological services in schools. Educational personnel also will need to be trained in the effect of chronic disease on the daily functioning of children, with a focus on academic achievement and successful socialization. Such training efforts will promulgate additional services for children and adolescents with recurrent pain and it is hoped will stimulate research by pain experts and pediatric and school psychologists. It is hoped that greater collaboration will provide better clinical services, longneeded research, and public policies that advocate for these children and their families.

\section{References}

1. Power TJ, Blom-Hoffman J. The school as a venue for managing and preventing health problems: opportunities and challenges. In: Brown RT, ed. Handbook of Pediatric Psychology in School Settings. Mahwah, NJ: Erlbaum; 2004, pp. 37-78.

2. Power TJ, Heathfield L, McGoey K, Blum NJ. Managing and preventing chronic health problems: school psychology's role. Sch Psychol Rev 1999;28:251-263.

3. Roberts MC, Brown KJ, Boles RE, Mashunkashey JO. Prevention of injuries: concepts and interventions for pediatric psychology in the schools. In: Brown RT, ed. Handbook of Pediatric Psychology in School Settings. Mahwah, NJ: Erlbaum; 2004, 65-80.

4. Walker LS. Helping the child with recurrent abdominal pain return to school. Pediatr Ann 2004;33:128-136.

5. Walker LS, Smith CA, Garber J, Van Slyke DA, Claar RL. The relation of daily stressors to somatic and emotional symptoms in children with and without recurrent abdominal pain. J Consult Clin Psychol 2001;69:85-91.

6. Tsao JCI, Glover DA, Bursch R, Ifewunigwe M, Zeltzer LK. Laboratory pain reactivity and gender: relationship to school nurse visits and school absences. J Dev Behav Pediatr 2002;23:217-224.

7. Tsao JC, Zeltzer LK. Sex differences in pain-related symptoms and self-initiated school nurse visits among pre-adolescents. J Pain Symptom Manage 2003;25: 472-480.

8. Walker LS, Greene JW. Negative life events and symptom resolution in pediatric abdominal pain patients. J Pediatr Psychol 1991;16:341-360.

9. Williams K, Chambers M, Logan S, Robinson D. Association of common health symptoms with bullying in primary school children. Br Med J 1996;313:17-19. 
10. Walker LS, Garber J, Greene JW. Psychosocial characteristics of recurrent childhood pain: a comparison of children with recurrent abdominal pain, organic illness, and psychiatric disorders. J Abnorm Child Psychol 1993;102:248-258.

11. Claar RL, Walker LS. Maternal attributions for the causes and remedies of their children's abdominal pain. J Pediatr Psychol 1999;24:345-354.

12. Walker LS. The evolution of research on recurrent abdominal pain: history, assumptions, and a conceptual model. In: McGrath PJ, Finley GA, eds. Progress in Pain Research and Management. Vol. 13. Seattle, WA: IASP Press; 1999:141-172.

13. Zeltzer LK, Bush JP, Chen E, Riveral A. A psychobiologic approach to pediatric pain: part I. History, physiology, and assessment strategies. Curr Prob Pediatr 1997;27:255-258.

14. Larsson B, Carlson J. A school-based, nurse-administered relaxation training for children with chronic tension-type headache. J Pediatr Psychol 1996;21:603-614.

15. American Psychiatric Association. Diagnostic and Statistical Manual of Mental Disorders. 4th ed. Washington, DC: American Psychiatric Association; 1994.

16. Levy RL, Whitehead WE, Van Korff MR, Feld AD. Intergenerational transmission of gastrointestinal illness behavior. Am J Gastroenterol 2000;95:451-456.

17. Walker LS, Claar RL, Garber J. Social consequences of children's pain: when do they encourage symptom maintenance? J Pediatr Psychol 2002;27:689-698.

18. Walker LS, Johnson WS. Recurrent abdominal pain and functional gastrointestinal disorders in the school setting. In: Brown RT, ed. Handbook of Pediatric Psychology in School Settings. Mahwah, NJ: Erlbaum; 2004, pp. 299-312.

19. Larsson B, Melin L. Chronic headaches in adolescents: treatment in a school setting with relaxation training as compared with information-contact and self-registration. Pain 1986;25:325-336.

20. Larrson B, Melin L, Lamminen M, Ulstedt F. A school-based treatment of chronic headaches in adolescents. J Pediatr Psychol 1987;12:553-566.

21. Brown RT. Introduction: changes in the provision of health care to children and adolescents. In: Brown RT, ed. Handbook of Pediatric Psychology in School Settings. Mahwah, NJ: Erlbaum; 2004, pp. 1-19.

22. Perrin JM, Ireys HT. The organization of service for chronically ill children and their families. Ped Clin North Am 1984;31:235-257.

23. Thompson RJ Jr, Gustafson KE, Hamlet KW, Spock A. Stress, coping, and family functioning in the psychological adjustment of mothers of children and adolescents with cystic fibrosis. J Pediatr Psychol 1992;17:573-585. 